\title{
Supporting Information: Spatiotemporally programmable metasurfaces via viscoelastic shell snapping
}

\author{
Yuzhen Chen ${ }^{1}$, Tianzhen $\operatorname{Liu}^{1}$, and Lihua $\operatorname{Jin}^{1}$ \\ ${ }^{1}$ Affiliation not available
}

December 22, 2021

\begin{abstract}
Supplementary Text 1: Material modeling and characterization We used the following incompressible neo-Hookean material model to define the instantaneous constitutive behavior of the shells, $=\operatorname{tr}-3$, (S1) where $\mathrm{W}$ is the strain energy density function, $\mu$ is the shear modulus, $\mathrm{F}$ is the deformation gradient tensor. To describe the viscoelastic behavior of the shells, Prony series were used and the shear modulus $\mu$ can be expressed as $=1-[?] 1-/$, (S2) where $\mu 0$ is the instantaneous shear modulus, $\mathrm{n}$ is the number of the series terms, is the dimensionless relaxation modulus, $t$ is the time, and $\tau i$ is the relaxation time constant. Here we characterize the viscoelastic properties of the silicone rubber (Dragon SkinTM30) and urethane rubber (VytaFlexTM 20). We modeled their viscou
\end{abstract}

\section{Hosted file}

20211220_manuscript_final_SI.pdf available at https://authorea.com/users/452194/ articles/550347-supporting-information-spatiotemporally-programmable-metasurfaces-viaviscoelastic-shell-snapping

\section{Hosted file}

movie_s1.mp4 available at https://authorea.com/users/452194/articles/550347-supportinginformation-spatiotemporally-programmable-metasurfaces-via-viscoelastic-shell-snapping

\section{Hosted file}

movie_s2.mp4 available at https://authorea.com/users/452194/articles/550347-supportinginformation-spatiotemporally-programmable-metasurfaces-via-viscoelastic-shell-snapping

\section{Hosted file}

movie_s3.mp4 available at https://authorea.com/users/452194/articles/550347-supportinginformation-spatiotemporally-programmable-metasurfaces-via-viscoelastic-shell-snapping 